\section{Ratio of Loading Dye to PCR Product Affects Sensitivity of Single- Stranded Conformational Polymorphism Analysis}

\author{
BioTechniques 34:1168-1170 (June 2003)
}

Rifampin resistance in Mycobacterium tuberculosis involves missense mutations, small insertions, or deletions (Table 1 and References 1 and 2) in a well-characterized region of the $r р о B$ gene encoding the $\beta$-subunit of the RNA polymerase (2). These mutations have been found in more than $97 \%$ of rifampin-resistant $M$. tuberculosis strains (3) and are located in the 81-bplong region of the rpoB gene. Singlestranded conformational polymorphism (SSCP) is a method that can detect these kind of mutations and has been evaluated as a potential tool for the genotypic detection of rifampin-resistant $M$. $t u$ berculosis by several authors (1,4-6).

Different parameters can affect the mobility shift in SSCP. These are temperature, ionic strength, the composition of the gel, and additives (7). The type of base change, base composition, and the size of the DNA fragment (8) are other important parameters that play a role in the sensitivity of SSCP. Here we report the enhanced sensitivity of SSCP by decreasing the loading dye to PCR product ratio.

A 305-bp fragment of the $r p o B$ gene (2) of rifampin-resistant M. tuberculosis strains, which spans the region of 81 $\mathrm{bp}$, was amplified and subjected to SSCP analysis. $\mathrm{H}_{37} \mathrm{Rv}$ strain (reference no. 27294; ATCC, Manassas, VA, USA), which is sensitive to rifampin, was used as control. The following SSCP conditions were used: $20^{\circ} \mathrm{C}$, with cooling provided by a water jacket; 200 $\mathrm{V}$; $0.5 \times$ TBE buffer (45 mM Tris, $\mathrm{pH}$ 8.3, $45 \mathrm{mM}$ boric acid, $1 \mathrm{mM}$ EDTA at $25^{\circ} \mathrm{C}$ ) and $10 \%$ acrylamide gel (crosslinking ratio $=3 \%$; total acrylamide percentage $=30 \%$ ). A MightySmall SE 260 (Hoefer Scientific Instruments, San Francisco, CA, USA) vertical slab gel unit was used $(100 \times 105 \times 0.75 \mathrm{~mm}$ gels). The $7-\mu \mathrm{L}$ PCR product was mixed with $1,3.5$, or $7 \mu \mathrm{L}$ SSCP load-

Table 1. Sequence Analysis of the rpoB Gene of Rifampin-Resistant M. tuberculosis Strains

\begin{tabular}{|lcc|}
\hline \multicolumn{2}{|c|}{ Strains } \\
\hline Missense mutation & $59(89.4 \%)$ & $98(89.1 \%)$ \\
Ser531Leu mutation* & $31(47.0 \%)$ & $46(41.8 \%)$ \\
His526Tyr mutation* & $8(12.1 \%)$ & $20(18.2 \%)$ \\
Two point mutations & $4(6.0 \%)$ & $2(1.8 \%)$ \\
Insertion (3 bp) & - & $1(0.9 \%)$ \\
Deletion (3 bp) & $1(1.5 \%)$ & $1(0.9 \%)$ \\
No mutation & $2(3.0 \%)$ & $8(7.3 \%)$ \\
Total & 66 & 110 \\
These data are from References 1 and 2, together with the two most frequent \\
mutations (indicated by *).
\end{tabular}

ing dye [(95\% formamide; Serva, Heidelberg, Germany), 20 mM EDTA (Merck, Darmstadt, Germany), 0.25\% bromphenol blue, and $0.25 \%$ xylen cyanol (both from Serva)], boiled for 5 min, placed on ice, loaded onto the gel, and electrophoresed for $120 \mathrm{~min}$. The gels were stained by silver staining. Figure 1 shows that, by varying the loading dye to PCR product ratio from 1:7 to $1: 1$, we produced different SSCP patterns in the $\mathrm{H}_{37} \mathrm{Rv}$ strain on given electrophoretic conditions. Multiple bands that were seen at a 1:7 ratio were reduced to three bands at a 1:1 ratio, the fastest being double-stranded, renaturated DNA. To compare the sensitivity of SSCP on these two conditions, a sample of 39 rifampin-resistant $M$. tuberculosis strains collected in Serbia was used. The electrophoresis time was $120 \mathrm{~min}$ for the samples mixed with 1 $\mu \mathrm{L}$ loading dye and $240 \mathrm{~min}$ for those mixed with $7 \mu \mathrm{L}$ loading dye. SSCP with a $1: 7$ ratio, compared to a $1: 1$ ratio, detected mutations in $10.3 \%$ more of the strains. Figure 2 shows examples of SSCP profiles for strains for which the change in electrophoretic mobility was detected only with the 1:7 loading dye to PCR product ratio. Multiple bands contributed to the informativeness of SSCP (Figure 2B). Especially, the change in mobility was frequently seen for the band nearest the renaturated DNA, the band that did not appear when 1:1 ratio was used. The nature of this band is unknown. Considering the position of the band in the gel, we can assume that a decreased amount of denaturant formamide allowed some kind of reannealing to occur. Because the
PCR product was not purified before SSCP analysis, excess primers may reanneal with ssDNA prior to entry into the gel. If this is the case, then samples that do not have this band (Figure 2B, lanes 1 and 3) might have mutations that are unfavorable for the reannealing of the primer, in spite of the decreased amount of formamide. In those strains that show a shift of this band, compared to $\mathrm{H}_{37} \mathrm{Rv}$ (Figure 2B, lanes 5 and 6), the mutation may act in such a way that reannealing still occurs but is accompanied with a change in conformation and electrophoretic mobility of that conformer. We sequenced a 81-bp-long region of the $r p o B$ gene of one strain that showed small changes in the SSCP profile on a 1:1 dilution and a shift in the band nearest the dsDNA on a 7:1 dilu-

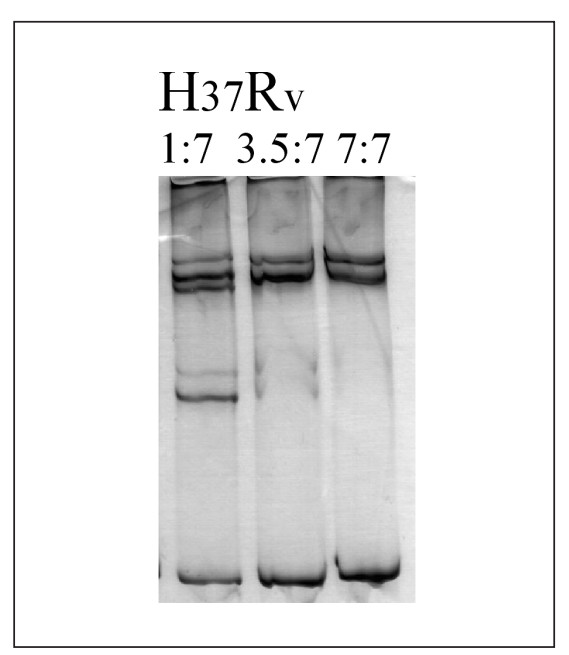

Figure 1. Change in SSCP profiles with increasing dilutions of PCR product. The 7- $\mathrm{PL}$ PCR product of $M$. tuberculosis $\mathrm{H}_{37} \mathrm{Rv}$ was mixed with either $1,3.5$, or $7 \mu \mathrm{L}$ loading dye. 


\section{Benchmarks}

A
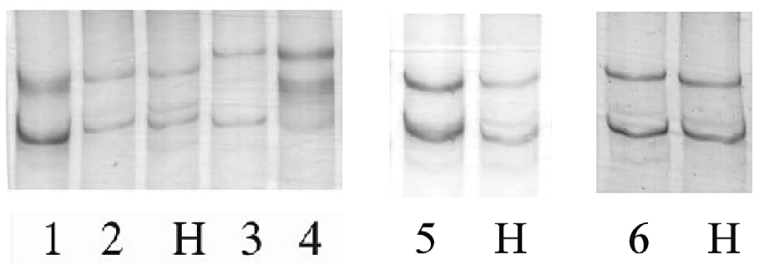

B

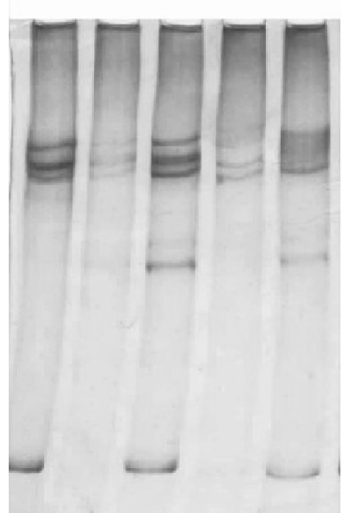

$5 \mathrm{H}$

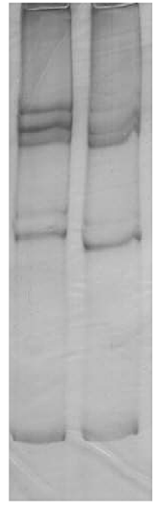

$6 \mathrm{H}$

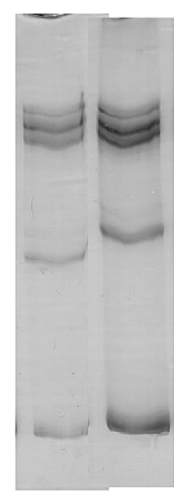

Figure 2. Comparison of SSCP analysis for two electrophoretic conditions. (A) A 1:1 loading dye to PCR product ratio. (B) A 1:7 loading dye to PCR product ratio. $\mathrm{H}_{37} \mathrm{Rv}$ was used as control (lane H). Rifampin-resistant $M$. tuberculosis strains with mobility shift detected only with the 1:7 ratio (lanes 1,5 , and 6). The low yield of PCR product, which is clearly visible on SSCP with the 1:1 ratio, is barely visible on the 1:7 ratio (wild-type SSCP profile on both conditions, lane 2; shift detected on both conditions, lane 3). Blurred band on both dilutions of the PCR product (lane 4).

tion of the PCR product. A $\mathrm{C} \rightarrow \mathrm{T}$ transition was found at position 526. The same transition, but at position 531, was found in another strain that we sequenced that had wild-type SSCP profiles on both dilutions of the PCR product. This mutation (Table 1) was reported to make only subtle changes in migration on the mutation detection enhancement gel (4) and, obviously, leads to very small changes in conformation of ssDNA, which did not cause any changes in electrophoretic mobility on our SSCP conditions. Since (in-frame) insertions and deletions are very rare in this fragment of the rроB gene of rifampin-resistant $M$. tuberculosis strains (Table 1), it is very possible that most of the other strains, if not all of them, including those $10.3 \%$, are with missense mutations. It should also be mentioned that, for two patients, SSCP analysis was performed for two isolates. In both cases, the SSCP analysis of the second isolate showed the same patterns as the first isolate that had been obtained several months earlier (see Figure 2, lane 6).

Hongyo et al. (9) studied the impact of loading solutions on SSCP. They proposed the 1:4 dilution of the PCR product and found that dilutions of less than 3:6 resulted in significant amounts of dsDNA and blurred bands in the SSCP gel. We found that the amount of dsDNA increased with increasing dilutions of the PCR product (Figure 1). Blurred bands were detected in two strains $(5.1 \%$, possibly with the same mutation), on both dilutions of the PCR product. The clear change in mobility was seen only when a 1:1 dilution was used (Figure 2). In addition, while multiple bands were barely visible when the amount of PCR product was smaller, the two bands on the 1:1 ratio were clearly visible (Figure 2 ).

In this collection of rifampin-resistant M. tuberculosis strains, the 1:7 loading dye to PCR product ratio enhanced SSCP sensitivity, compared to the 1:1 ratio, by $10.3 \%$. Therefore, decreasing the dilution of the PCR product should also be considered as a parameter that can increase the sensitivity of SSCP on certain electrophoretic conditions and enable the detection of some of the shifts that lacked when an equal ratio was used.

\section{REFERENCES}

1.Telenti, A., P. Imboden, F. Marchesi, D. Lowrie, S. Cole, M.J. Colston, L. Matter, K. Schopfer, et al. 1993. Detection of rifampicin-resistance mutations in Mycobacterium tuberculosis. Lancet 341:647-650.

2.Williams, D.L., C. Waguespack, K. Eisenach, J.T. Crawford, F. Portaels, M. Salfinger, C.M. Nolan, C. Abe, et al. 1994. Characterization of rifampin resistance in pathogenic mycobacteria. Antimicrob. Agents Chemother. 38:2380-2386.

3.Telenti, A. 1998. Genetics of drug resistant tuberculosis. Thorax 53:793-797.

4.Felmlee, T.A., Q. Liu, A.C. Whelen, D. Williams, S.S. Sommer, and D.H. Persing. 1995. Genotypic detection of Mycobacterium tuberculosis rifampin resistance: comparsion of single-strand conformation polymorphism and dideoxy fingerprinting. J. Clin. Microbiol. 33:1617-1623.

5.Kim, B.-J., S.-Y. Kim, B.-H. Park, M.-A. Lyu, I.-K. Park, G.-H. Bai, S.-J. Kim, C.-Y Cha, et al. 1997. Mutations in the $r p o B$ gene of Mycobacterium tuberculosis that interfere with PCR-single-strand conformation polymorphism analysis for rifampin susceptibility testing. J. Clin. Microbiol. 35:492-494.

6.Scarpellini, P., S. Braglia, A.M. Brambilla, M. Dalessandro, P. Cichero, A. Gori, and A. Lazzarin. 1997. Detection of rifampin resistance by single-strand conformation polymorphism analysis of cerebrospinal fluid of patients with tuberculosis of the central nervous system. J. Clin. Microbiol. 35:2802-2806.

7.Ravnik-Glavač, M., D. Glavač, and $M$. Dean. 1994. Sensitivity of single-strand conformation polymorphism and heteroduplex method for mutation detection in the cystic fibrosis gene. Hum. Mol. Genet. 3:801-807.

8.Sarkar, G., H.-S. Yoon, and S.S. Sommer 1992. Screening for mutations by RNA single-strand conformation polymorphism (rSSCP): comparison with DNA-SSCP. Nucleic Acids Res. 20:871-878.

9.Hongyo, T., G.S. Buzard, R.J. Calvert, and C.M. Weghorst. 1993. "Cold SSCP": a simple, rapid and non-radioactive method for optimized single-strand conformation polymorphism analyses. Nucleic Acids Res. 21:3637-3642.

Address correspondence to Dr. Zorica Lepsanovic, Institute of Preventive Medicine, Military Medical Academy (VMA), Crnotravska 17, 11000 Belgrade, Yugoslavia.e-mail: zorilep@eunet.yu

Received 29 January 2003; accepted 7 April 2003.

\section{Jelena Tesic, Zorica Lepsano- vic, and Veljko Mirovic Military Medical Academy Belgrade, Yugoslavia}

